\title{
An Estimation Algorithm of the Point Spread Function based on Singular Value Decomposition and Telemetry Prior Information
}

\author{
Wei Wang ${ }^{1, a}$ \\ ${ }^{1}$ The Chinese People's Liberation Army Unit 91550, Dalian, 116023, China \\ aemail: WeiWangDL@163.com
}

Keywords: Telemetry Prior Information; Point Spread Function; Singular Value Decomposition

\begin{abstract}
For the research of restoring the atmospheric turbulence-degraded images, an estimation algorithm of the point spread function based on singular value decomposition and telemetry prior information is proposed. The experiment shows image restoration effect of the proposed estimation algorithm.
\end{abstract}

\section{Introduction}

By the influence of atmospheric turbulence, the refractive index of reflected light changes with time and space random variation, light propagation direction and phase are dithering, which make the proliferation of point intensity distributing [1]. These posed the object measure quite a bit of difficulties, so it is necessary to do image recovery processing. Atmospheric turbulence-degraded image restoration is a difficult task, as the key part of image restoration, estimation of the point spread function (PSF) is very important [2]. The estimation of point spread function can be classified into two methods. The first class methods achieve PSF and restored algorithm as distinguished processes, which will achieve PSF without real image , then leverage classic restored algorithm to restore degraded image [3] [4]. The second class methods combine PSF recognition and restored algorithm to achieve restored image and PSF at the same time [5] [6].

This paper introduces a new algorithm, to use telemetry velocity data of a moving object, as telemetry prior information, and combine with singular value decomposition, so as to estimate $\mathrm{s}$ point spread function from turbulence-degraded image. Calculate position information separately from turbulence-degraded image and restoration image, then compares the calculation results with GPS position information of a moving object. The experiment shows image restoration effect of this algorithm.

\section{Design of the Algorithm}

For matrix B, can be show as sum function of singular values:

$$
B=\sum_{r=1}^{R} s_{r} u_{r} v_{r}^{H}
$$

In the type: $u_{r}$ is left singular value vector; $v_{r}$ is right singular value vector; $S$ is singular values matrix of $\mathrm{B} ; s_{r}$ is the $r$ th singular value in diagonal line of $S$; $R$ is rank of $B$. The discrete form of degraded image can export:

$$
g(x, y)=\sum_{m=1}^{2 K+1} \sum_{n=1}^{2 L+1} h(m, n) f(m-x, n-y)+n(x, y)
$$

Ignoring the influence of noise, the degraded process of original image can be interpreted as below, pixels $(x, y)$ of original image $(K, L)$ move position $(m, n)$, then multiple weighting function $h(m, n)$, summarize up, translation of image matrix on vertical direction reflects on left singular value vector, while translation on horizontal direction reflects on right singular value vector[7]. Type (2) can be rewrite:

$$
\sum_{r=1}^{R_{g}} s_{r g} u_{r g} v_{r g}{ }^{T}=\sum_{r=1}^{R_{f}} s_{r f} \sum_{m=1}^{2 K+1} \sum_{n=1}^{2 L+1} h(m, n) g_{r f m} v_{r f n}{ }^{T}
$$


Detailed algorithm is demonstrated as below.

Step1:

Combine the PSF form of first order singular value decomposition with type (3), get the same rank to both equations[8], get type (4):

$$
\sum_{r=1}^{R} u_{r g}=\sum_{r=1}^{R} u_{r f} * u_{h}, \quad \sum_{r=1}^{R} v_{r g}=\sum_{r=1}^{R} v_{r f} * v_{h}
$$

In the type, * is the linear convolution.

Step2:

Structure exponential model of mean energy spectrum density with singular value vector of ideal image, as type (5):

$$
s_{u f}=s_{v f}=\operatorname{DFT}\left(k_{R}^{|n|}\right)
$$

In the type, $s_{u f}$ and $s_{v f}$ are mean energy spectrum density of left-right $R$ th rank singular value vector respectively. $k_{R}$ is the mean of autocorrelation coefficient with $R$ rank singular value vector. Get coefficient $n$ as type (6), $V t$ is unitary telemetry velocity data of a moving object, $a$ is amplitude adjust factor form statistical velocity data during the flight of moving object. $b$ is sensibility adjust factor by manual setting.

$$
n=a \mathrm{~g} V t \mid /\left(1+e^{b \mathrm{~d} V t \mid}\right)
$$

The choice of restructuring rank $R$ in amplitude estimation is determined by the second derivative of the cumulative sum function of singular values. Based on second derivative curve of the cumulative sum function of singular values, to observe the change of singular values, hence to choose the rank of estimation of point spread function, normally, the rank is small, therefore, related singular value vector, SNR is relatively high, and impact of noise is low. Cumulative sum function of singular values shows in below type[9].

$$
c(i)=\sum_{r=1}^{i} s_{r}, i=1,2, \ldots N
$$

Step3:

Get amplitude squares of type (5), and show PSF as singular value decomposition.

$$
\sum_{r=1}^{R} s_{r u g}=\sum_{r=1}^{R} s_{r u f} g_{u h}, \quad \sum_{r=1}^{R} s_{r v g}=\sum_{r=1}^{R} s_{r v f} g_{v h}
$$

In the type, $s_{r u g}$ and $s_{r v g}$ are energy spectrum density of left-right $R$ th rank singular value vector in degraded image respectively. $s_{r u f}$ and $s_{r v f}$ are energy spectrum density of left right Rth rank singular value vector in ideal image respectively. $s_{u h}$ and $s_{v h}$ is the spectrum of PSF. $R$ is restructuring rank.

Step4:

Get the amplitude estimation of first order singular value vector spectrum as below.

$$
u_{h}=\sqrt{\sum_{r=1}^{R} s_{r u g} / R g_{u f}}, \quad v_{h}=\sqrt{\sum_{r=1}^{R} s_{r v g} / R g_{v f}}
$$

Step5:

Set the phase of the first order singular value vector spectrum of PSF as 0. Detect spectral amplitude values above 0 , do $180^{\circ}$ phase translation. Do coupling the amplitude estimation of left and right singular value with phase, conduct inverse Fourier transform to first order singular value of left and right, determine the boundary, then reconstruct and regress to get estimation of PSF.

\section{Experiment and Results}

In the experiment, an atmospheric turbulence-degraded image of moving object is shown in Figure 1 (a). Set amplitude adjust factor $a=2.56$ based on the statistical velocity data during the flight of moving object. Set sensibility adjust factor $b=0.15$. The cumulative sum curve of energy spectrum density with left-right singular value vector is shown in Figure 2. Restoration of the 
degraded image is achieved by inverse filtering based on the PSF from the proposed estimation algorithm in this paper, the result shown in Figure 1 (b).

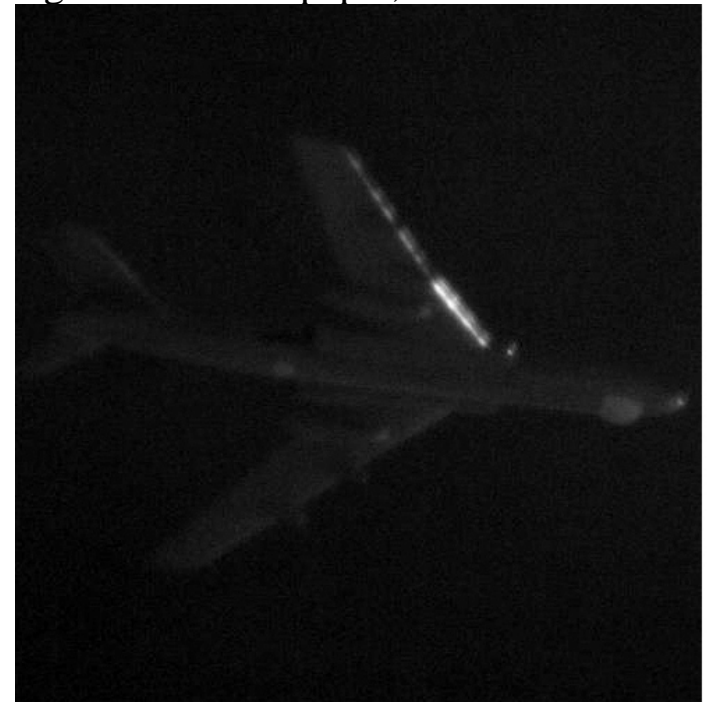

(a) atmospheric turbulence-degraded image

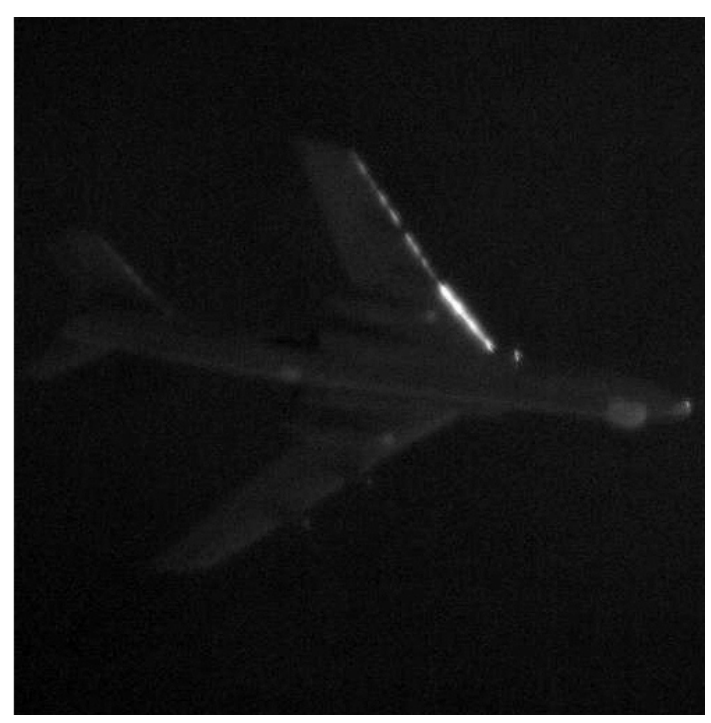

(b) restoration of the degraded image

Fig.1. The experimental results

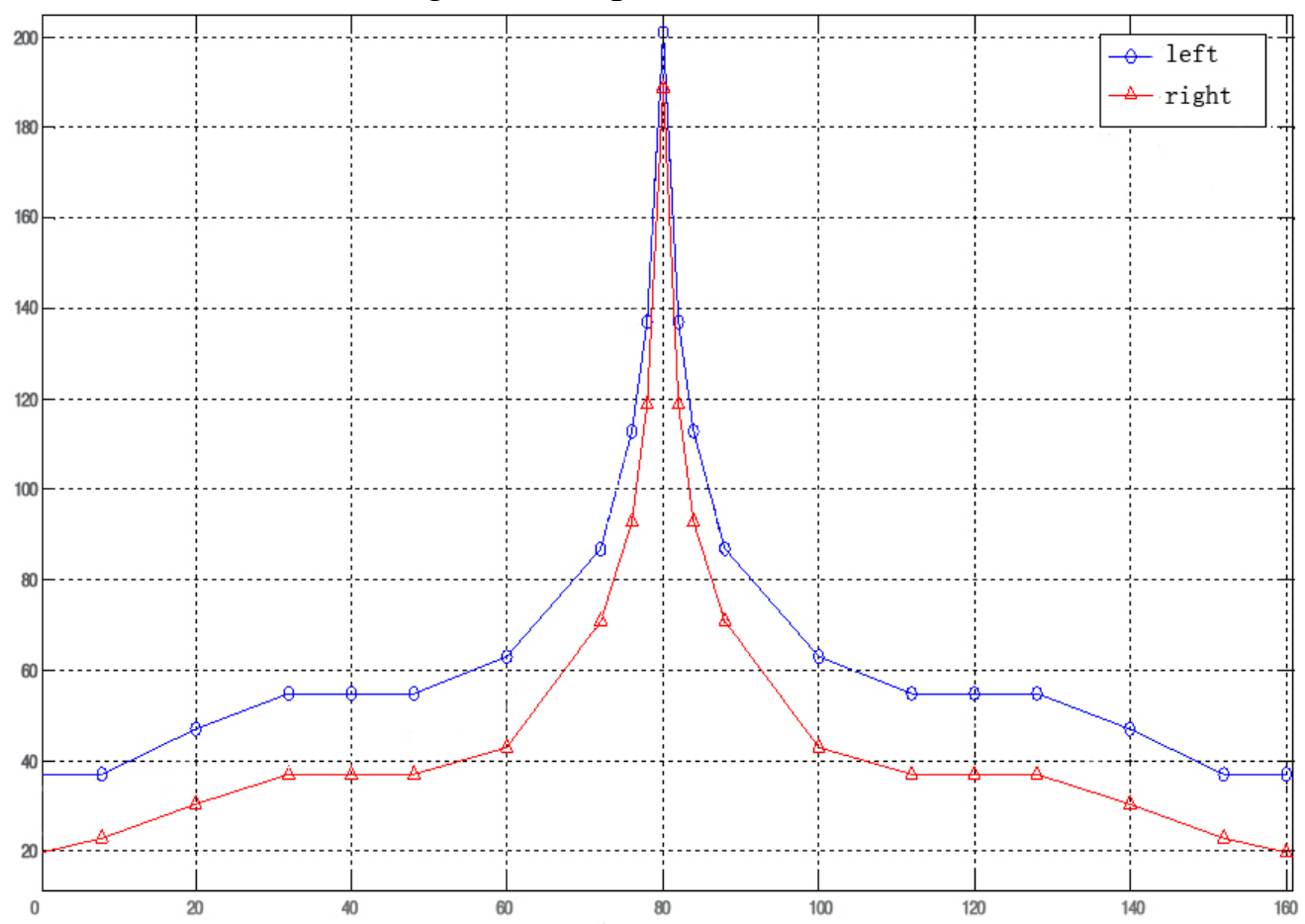

Fig.2. The cumulative sum curve of energy spectrum density with left-right singular value vector

\section{Conclusion}

As shown in Figure 1, the estimation algorithm of the point spread function based on singular value decomposition and telemetry prior information can obtain valuable PSF for restoration of the atmospheric turbulence-degraded image. The experiment proves the benefits of algorithm proposed in this paper.

\section{References}

[1] Narasimhan S.G, Nayar S.K. Contrast restoration of weather degraded images[J]. IEEE Transactions on Pattern Analysis and Machine Intelligence,2003, 25(6),713-724 . 
[2] Li Da-long. Restoration of atmospheric turbulence degraded video using kurtosis minimization and motion compensation[D]. Georgia:Georgia Institute of Technology, 2007,1-32.

[3] Jiang Ming, Wang Ge. Development of blind image deconvolution and its applications[J]. Journal of X-Ray Science and Technology, 2003 , 11:13-19.

[4] Mahesh B. Chappalli, N. K. Bose. Enhanced Biggs-Andrews asymmetric iterative blind deconvolution [J]. Multidim Syst Sign Process ,2006,17:151-175.

[5] Ying Liang, Changhui Rao, Mei Li,et al. Iterative blind deconvolution of adaptive optics images[J].Chinese Optics Letters,2006,4(4).

[6] Xiaochao Gan, Alan Wee-Chung Liew, Hong Yan. A POCS-Based constrained total least squares algorithm for image restoration[J]. J. Vis Commum. Image R.2006,1-18.

[7] R.Tan. Visibility in bad weather from a single image[J]. In IEEE Conference on Computer Vision and Pattern Recognition (CVPR’08), 2008,1-8.

[8] Kaiming He, Jian Sun, Xiaoou Tang. Single image haze removal using dark channel prior[J]. CVPR, 2009, 1956-1963.

[9] Jean-Philippe, Tarel. Visibility Restoration from a Single Color or Gray Level Image[J], International Conference on Computer Vision,2009. 